\title{
Transport properties of single-walled carbon nanotube transistors after gamma radiation treatment
}

S. A. Vitusevich, V. A. Sydoruk, M. V. Petrychuk, B. A. Danilchenko, N. Klein, A. Offenhäusser, A. Ural, and G. Bosman

Citation: Journal of Applied Physics 107, 063701 (2010);

View online: https://doi.org/10.1063/1.3340977

View Table of Contents: http://aip.scitation.org/toc/jap/107/6

Published by the American Institute of Physics

\section{Articles you may be interested in}

Radiation effects in single-walled carbon nanotube papers

Journal of Applied Physics 107, 014316 (2010); 10.1063/1.3268470

Effect Of Gamma-Irradiation on Single-Wall Carbon Nanotube Paper

AIP Conference Proceedings 685, 143 (2003); 10.1063/1.1628005

Schottky barriers in carbon nanotube-metal contacts

Journal of Applied Physics 110, 111101 (2011); 10.1063/1.3664139

Ion irradiation of electronic-type-separated single wall carbon nanotubes: A model for radiation effects in nanostructured carbon

Journal of Applied Physics 112, 034314 (2012); 10.1063/1.4739713

Generation of continuous and pulsed diagnostic imaging x-ray radiation using a carbon-nanotube-based fieldemission cathode

Applied Physics Letters 81, 355 (2002); 10.1063/1.1492305

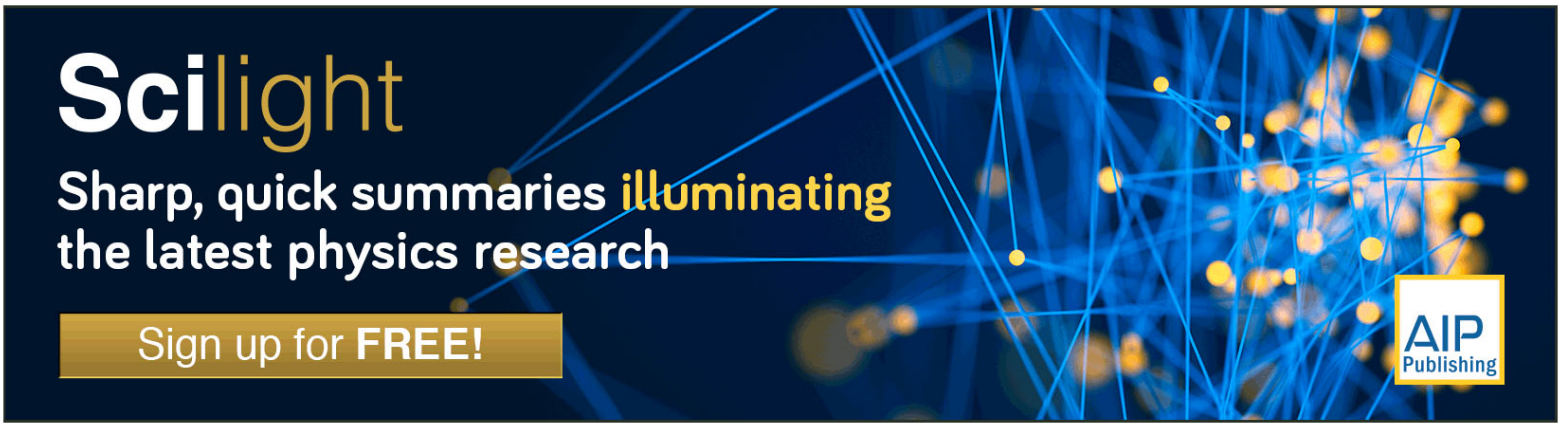




\title{
Transport properties of single-walled carbon nanotube transistors after gamma radiation treatment
}

\author{
S. A. Vitusevich, ${ }^{1, a)}$ V. A. Sydoruk, ${ }^{1}$ M. V. Petrychuk, ${ }^{2}$ B. A. Danilchenko, ${ }^{3}$ N. Klein, ${ }^{1}$ \\ A. Offenhäusser, ${ }^{1}$ A. Ural, ${ }^{4}$ and G. Bosman ${ }^{4}$ \\ ${ }^{1}$ Institute of Bio- and Nanosystems and Jülich-Aachen Research Alliance for Future Information Technology \\ (JARA-FIT), Forschungszentrum 52425 Jülich, Germany \\ ${ }^{2}$ Taras Shevchenko National University, Kiev, 01033, Ukraine \\ ${ }^{3}$ Institute of Physics, NASU, Kiev, Ukraine \\ ${ }^{4}$ Department of Electrical and Computer Engineering, University of Florida, Gainesville Florida 32611, \\ USA
}

(Received 7 August 2009; accepted 30 January 2010; published online 16 March 2010)

\begin{abstract}
Single-walled carbon nanotube field-effect transistors (CNT-FETs) were characterized before and after gamma radiation treatment using noise spectroscopy. The results obtained demonstrate that in long channel CNT-FETs with a length of $10 \mu \mathrm{m}$ the contribution of contact regions can be neglected. Moreover, radiation treatment with doses of $1 \times 10^{6}$ and $2 \times 10^{6}$ rad allows a considerable decrease parallel to the nanotube parasitic conductivity and even the shift region with maximal conductivity to the voltage range of nearly zero gate voltage that improves the working point of the FETs. The Hooge parameters obtained before and after gamma radiation treatment with a dose of $1 \times 10^{6} \mathrm{rad}$ are found to be about $5 \times 10^{-3}$. The parameters are comparable with typical values for conventional semiconductors. (C) 2010 American Institute of Physics.
\end{abstract}

[doi:10.1063/1.3340977]

\section{INTRODUCTION}

One-dimensional nanostructures such as carbon nanotubes (CNTs) have attracted considerable research efforts owing to their strong application potential for high-speed nanosize electronics and biosensors utilizing their superior electronic properties. ${ }^{1}$ CNTs are chemically stable and electrically conducting tubes. Depending on the chirality, they can be either metallic or semiconducting, however, only semiconducting-type CNTs can be controlled by the gate voltage. At the same time, this different behavior with gate voltage allows us to study conductivity in semiconductor and metallic tubes separately-just by using the appropriate gate voltage. A CNT is a one-atom-thick crystal layer, therefore, transport phenomena of CNTs are strongly sensitive to the surface conditions that can be used for the development of chemosensors and biosensors. Moreover, not only surface phenomena determine transport properties but also the performance of nanotube transistors is limited by the strong influence of the Schottky barriers at the metal-CNT interface. These contact regions play an essential role in the formation of the transport mechanisms of CNT-based field-effect transistors (FETs) with small lengths of the channel. ${ }^{2}$ Typically, the formation of Schottky barrier is registered at the metal/ semiconductor interface as a result of combined effect of metal work function and Fermi level pinning by surface states. ${ }^{3}$ Decreasing the Schottky barriers and in the ideal case obtaining ohmic contact at the contact regions will allow the intercontact distances to be reduced simultaneously using the advantages of channel transport phenomena with different

\footnotetext{
${ }^{a}$ Author to whom correspondence should be addressed. Electronic mail: s.vitusevich@fz-juelich.de. On leave from Institute of Semiconductor Physics, NASU, Kiev, Ukraine.
}

surface modified conditions in CNTs. Therefore, the study, modification, and optimization of conductivity in CNT-FETs have recently become an important research topic. One of the most effective methods for modifying material properties and interface states is gamma radiation treatment. However, for the case of CNTs such investigations are limited by a sufficiently high dose of gamma irradiation of $5 \times 10^{6}$ rad. $^{4-6}$ At the same time, very interesting effects of treatment are observed after gamma irradiation at small doses for conventional semiconductor devices. ${ }^{7}$ During increasing accumulation dose, regions of certain radiation doses can be found, where the structural and electrical parameters of materials can be improved due to radiationstimulated diffusion process and the structural ordering of native defect structures and strain relaxation effect. ${ }^{8}$

In the present work, we investigated the influence of $\gamma$-radiation with several small doses of radiation treatment on transport properties of single-walled CNT-FETs by using a ${ }^{60} \mathrm{Co}$ source. Noise spectroscopy is used to extract additional important information about the mechanisms of transport formation in the multilayer structure of FETs before and after gamma radiation treatment.

\section{EXPERIMENTAL DETAILS}

FETs with backside gate configuration were used. Such a configuration allows us to use a simple and convenient method of studying transport phenomena in an ensemble of CNTs. Structures under study consist of two Mo contacts deposited at a distance of $10 \mu \mathrm{m}$ on $\mathrm{SiO}_{2}$ on the top of heavily doped $\mathrm{Si}$ substrate, where the $\mathrm{Si}$ substrate is used as a back gate. The catalyst regions were processed as $5 \mu \mathrm{m}$ $\times 2 \mathrm{~mm}$ strips. Nanotubes were grown using an aluminasupported Fe/Mo catalyst placed on the contacts. The cata- 

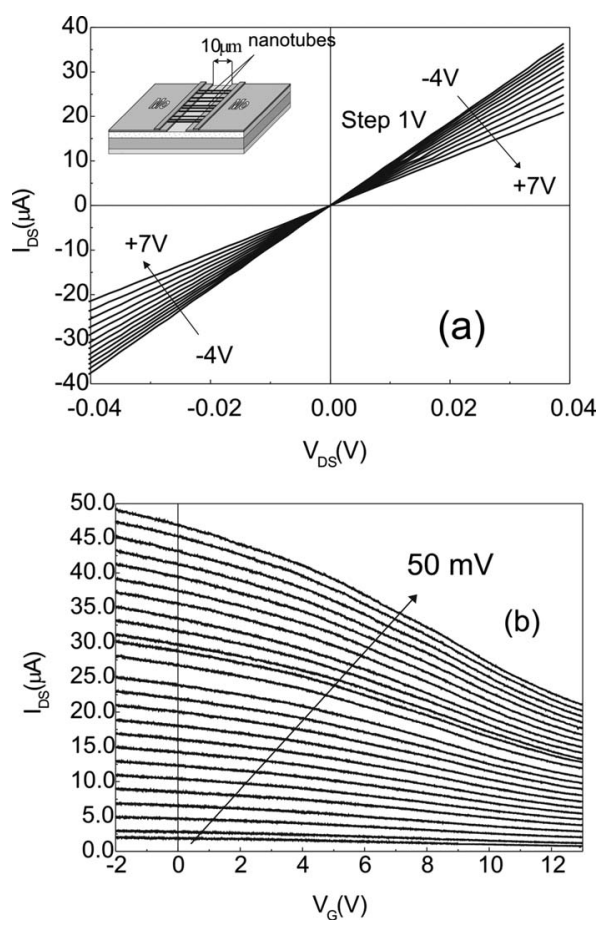

FIG. 1. I-V characteristics of the investigated CNT-FETs measured before gamma radiation treatment: (a) output characteristics measured at different gate voltages with $1 \mathrm{~V}$ step. Inset: schematic of the investigated CNT-based FETs; and (b) transfer characteristics measured at different drain-source voltages in the range from $0 \mathrm{~V}$ to $50 \mathrm{mV}$.

lyst strips were positioned $5 \mu \mathrm{m}$ away from the edges of the Mo electrodes. The structure under study is shown schematically in the inset to Fig. 1(a). CNTs were grown at $900{ }^{\circ} \mathrm{C}$ for $2 \mathrm{~min}$ under $720 \mathrm{ml} / \mathrm{min}$ of methane, $500 \mathrm{ml} / \mathrm{min}$ of hydrogen, and $12 \mathrm{ml} / \mathrm{min}$ of ethylene flow. Electric fields of the order of $1 \mathrm{~V} / \mu \mathrm{m}$ were used to control the orientation of the growth. ${ }^{9}$ In the structures under test typically 500-600 nanotubes were found to be connected in parallel between the contacts using scanning electron microscope. Using parallel aligned CNTs it is possible to remove crossover among CNTs and considerably reduce noise level by removing the noise generated by tube-tube junctions. ${ }^{10}$ It will be shown below that such a structural design with a sufficiently large length between the contacts allows effective current control through the CNTs of FET structures rather than the contact resistance.

The diameters of the nanotubes were measured using an atomic force microscope and found to be about $1.3 \mathrm{~nm}$. The scanning electron beam microscope images were obtained demonstrating clear alignment in the direction of the electric field, perpendicular to the edges of the Mo electrodes. A standard isotope ${ }^{60} \mathrm{Co}$ source was used to emit characteristic gamma rays with a flux of $10^{2} \mathrm{rad} / \mathrm{s}$ and an energy of 1.2 $\mathrm{MeV}$. We measured the I-V characteristics of the FETs and the low-frequency noise spectra in the frequency range from $1 \mathrm{~Hz}$ to $100 \mathrm{kHz}$ before and after gamma radiation treatment.

\section{EXPERIMENTAL RESULTS AND DISCUSSION}

Figure 1(a) shows the output characteristics of the FET measured at different gate biases. The device displays symmetric behavior for positive and negative drain voltages. As can be seen from the figure, pinch-off is not reached in the device. This is related to the conductivity through the metallic CNTs and the conductance which cannot be controlled by the gate voltage. Typical transfer characteristics obtained before irradiation are shown in Fig. 1(b). The semiconducting CNTs at fixed drain-source voltage, $V_{\mathrm{DS}}$, display characteristic $p$-type behavior by decreasing current with increasing (from the negative to positive) gate voltage applied to the substrate. The maximum transconductance, $g_{m}$, measured at a drain-source bias of $50 \mathrm{mV}$ and gate-source bias of $9 \mathrm{~V}$ is found to be as high as $2.7 \mu \mathrm{S}$. The relatively low ratio of the current in off and on states is due to one third of metallic CNTs being arranged in parallel to two thirds semiconducting CNTs in the channel. Such switching behavior with applied gate voltage is widely adopted in analog rf systems where some voltage region is used around a fixed working point.

The effective mobility, $\mu$, of the CNT can be estimated using the following equation for long-channel FET at low drain-source voltage: ${ }^{11}$

$$
\mu=\frac{L_{\mathrm{DS}}^{2} g_{m}}{C_{G} V_{\mathrm{DS}}}
$$

with $C_{G}=2 \pi L_{\mathrm{DS}} \varepsilon_{r} \varepsilon_{0} / \ln (2 t / r)$, where $L_{\mathrm{DS}}$ is the transistor channel length, $V_{\mathrm{DS}}$ is the drain voltage, $\varepsilon_{r}$ is the dielectric constant of $\mathrm{SiO}_{2}, t$ is the thickness of the $\mathrm{SiO}_{2}$ layer, and $r$ is the radius of a CNT. Using $\varepsilon_{r}=3.9, t=500 \mathrm{~nm}, V_{\mathrm{DS}}$ $=50 \mathrm{mV}, L_{\mathrm{DS}}=10 \mu \mathrm{m}$, and $r=1.3 \mathrm{~nm}$, we obtain $\mu=1.5$ $\times 10^{5} \mathrm{~cm}^{2} / \mathrm{V} \mathrm{s}$. Such a mobility value is characteristic of good quality CNTs. ${ }^{1}$

A small dose of gamma radiation treatment results in positive changes of the FET I-V characteristics. The increase in the current ratio in off and on states and shift in the threshold voltage in direction to zero voltage are registered. In addition, the influence of parasitic conduction channels on dc characteristics of the device decreases, as this will be shown below. The transfer characteristics of the FET measured after irradiation with a dose of $1 \times 10^{6} \mathrm{rad}$ are shown by dotted lines in Fig. 2(a). The current through the structure can be effectively controlled by the gate with the same amplitude of modulation recorded before radiation treatment. The transconductance of the FETs practically did not change [Fig. 2(a)] but the level of the current decreases. This difference in current measured before, $I_{\mathrm{DS} 0}$, and after irradiation, $I_{\mathrm{DSR}}$, is a linear function on drain voltage [Fig. 2(b)], which indicates that either some linear resistance was added in series to the nanotubes or a parallel resistance was removed by irradiation. The dotted line is a linear fit of difference in drain current on $V_{\mathrm{DS}}$

$$
\Delta I_{\mathrm{DS}}=-1.85 \times 10^{-4} V_{\mathrm{DS}}-8.48 \times 10^{-8} \approx-\frac{V_{\mathrm{DS}}}{5400}
$$

If we assume that a serial resistance, $R_{\text {add }}$, was added to the total resistance, $R_{T}$, by irradiation, then the current difference should be a function of gate voltage, $V_{G}$, 

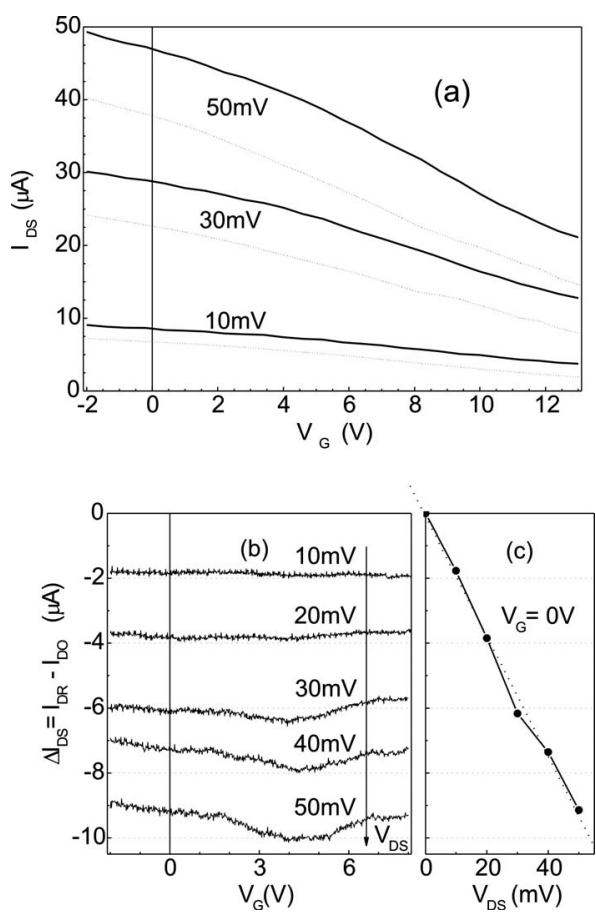

FIG. 2. Comparison of transfer characteristics of CNT-FETs measured before and after gamma radiation treatment with a dose of $1 \times 10^{6} \mathrm{rad}$ measured at different drain-source voltages: (a) solid lines-before irradiation, dotted lines-after irradiation; (b) dependences of the difference between currents after and before irradiation on gate voltage, $V_{G}$, at different drainsource voltages; and (c) difference between currents after and before irradiation as a function of drain-source voltage measured at $V_{G}=0 \mathrm{~V}$, dotted curves show calculated dependences.

$$
\begin{aligned}
\Delta I_{\mathrm{DS}}= & \frac{V_{\mathrm{DS}}}{R_{T}\left(V_{G}\right)+R_{\mathrm{add}}}-\frac{V_{\mathrm{DS}}}{R_{T}\left(V_{G}\right)}= \\
& -\frac{V_{\mathrm{DS}} \times R_{\mathrm{add}}}{\left[R_{T}\left(V_{G}\right)+R_{\mathrm{add}}\right] \times R_{T}\left(V_{G}\right)}=f\left(V_{G}\right) .
\end{aligned}
$$

Indeed I-V characteristics measured after $1 \times 10^{6} \mathrm{rad}$ dose demonstrate about the same effective control of the drain current at different gates, which indicates that the resistance of the contact regions are about the same after 1 $\times 10^{6} \mathrm{rad}$ dose of gamma rays.

If we assume that radiation removed a resistance parallel to the nanotubes, then the current difference should be independent of $V_{G}$

$$
\Delta I_{\mathrm{DS}}=\frac{V_{\mathrm{DS}}}{R_{T}\left(V_{G}\right)}-\left[\frac{V_{\mathrm{DS}}}{R_{T}\left(V_{G}\right)}+\frac{V_{\mathrm{DS}}}{R_{\text {add }}}\right]=-\frac{V_{\mathrm{DS}}}{R_{\mathrm{add}}} \neq f\left(V_{G}\right) .
$$

From experimental data [Fig. 2(b)] it is found that the decreasing of current is independent of gate voltage. Therefore, the difference between the drain currents before and after radiation treatment (which can be accounted for by the contribution of a parallel resistance of $5.4 \mathrm{k} \Omega$ ) is due to changes of the structure in the channel region but not due to changes in contact resistance.

Let us consider the ionization processes under the influence of gamma radiation with a small dose in our CNT-FET structure. It has been previously established that the main changes in the layered structure occurred in more resistive regions as result of changes introduced by the Compton effect. Therefore, we will consider gamma radiation processes starting from dielectric $\mathrm{SiO}_{2}$ layer grown on the top of the heavily doped $\mathrm{Si}$ substrate.

The effect of irradiation on metal-oxide-silicon FETs has been widely studied. ${ }^{12}$ The energy of ionization on silicon oxide has been estimated to be roughly $18 \mathrm{eV}$. The electrons generated by radiation have mobility of the order of $20 \mathrm{~cm}^{2} / \mathrm{V} \mathrm{s}$ at room temperature. The mobility of the holes is in the range of $10^{-4} \mathrm{~cm}^{2} / \mathrm{V} \mathrm{s}$. Although without an applied electric field the generated electron/hole pairs have a higher probability of recombining, the fraction of nonrecombined charge might nevertheless be up to $30 \%$ in the case of cobalt irradiation. In addition, the electric field applied between the source and drain sweeps the electrons out from the oxide rapidly in comparison with holes.

Gamma irradiation results in the trapping of holes in the $\mathrm{SiO}_{2}$ near the $\mathrm{Si} / \mathrm{SiO}_{2}$ interface and the creation of interface states at the $\mathrm{Si} / \mathrm{SiO}_{2}$ boundrary. ${ }^{13}$ The density of radiationinduced states of the first type of center is roughly equal to the density of radiation-induced interface states. The density of a radiation-induced second type of center was found to be of the same order of magnitude as that of holes trapped in the oxide and situated very near the $\mathrm{Si} / \mathrm{SiO}_{2}$ interface, all within about $10 \mathrm{~nm}$. A third type of centers is the nonbridging oxygen center. The radiation-induced positive charge (mainly holes) is transferred to the $\mathrm{Si} / \mathrm{SiO}_{2}$ interface by positive bias applied to the oxide layer with respect to substrate and is found to interact with precursors of the interface traps.

Therefore, the insulating oxide layer is the part of our device most sensitive to such ionization due to its high resistance so that conductivity is strongly sensitive to defects introduced by gamma radiation. The interface between the amorphous oxide and the crystalline silicon is generally deficient in oxygen, giving rise to dangling silicon bonds. These dangling bonds act as interface traps with energy levels within one or two atomic bond distances $(0.5 \mathrm{~nm})$ from the silicon lattice. High-quality $\mathrm{Si} / \mathrm{SiO}_{2}$ structures have preirradiation interface trap densities in the range of $10^{9}-10^{10}$ traps $/ \mathrm{cm}^{2}$. In the bulk of the oxide film, the silicon ionization and the interstitial oxygen donor centers are shown to be responsible for the radiation-generated positive space charge build-up (oxide charge) in thermally grown silicon oxide. ${ }^{14}$ Usually nonrecombined holes remain initially close to their points of creation and cause a negative shift in the flat band voltage and the threshold voltage. ${ }^{15}$ Holes created by gamma irradiation result in decreasing summary resistance.

In the investigated CNT-FET structures, we did not observe any shift in threshold voltage or decrease in the summary resistance after a small dose of gamma radiation of 1 $\times 10^{6} \mathrm{rad}$. Moreover, increasing summary resistance was registered. This fact demonstrates that the main changes in transport properties after gamma radiation treatment are related to channel conductivity determined by CNTs. The origin of this modification can be explained by a slight modification of surface atom ordering in CNT due to the introduction of vacancies and interstitial point defects. The results of noise spectroscopy give additional important infor- 

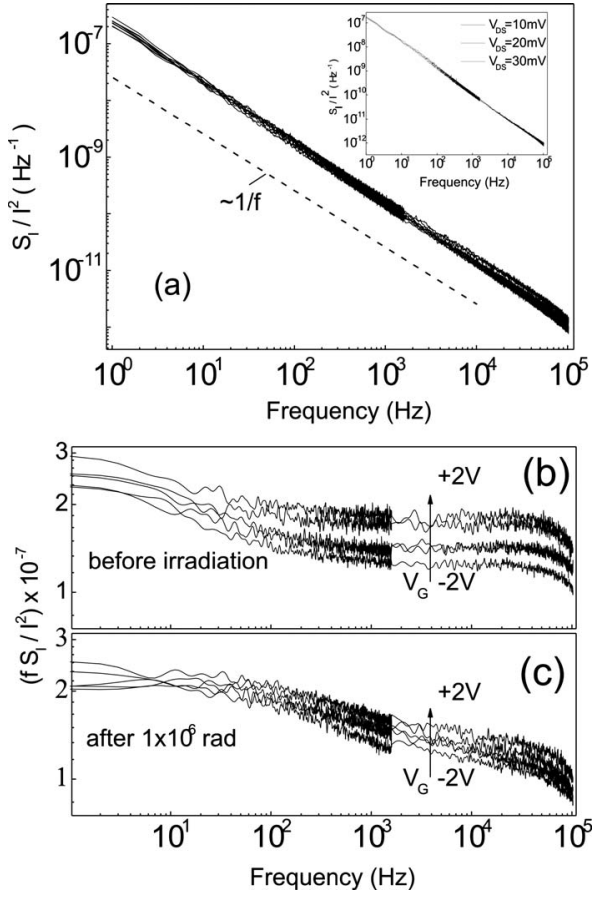

FIG. 3. Normalized current noise power spectra of CNT-FETs (a) measured before and after irradiation at different $V_{G}$ in the range from -2 to $2 \mathrm{~V}$ with step $1 \mathrm{~V}$ and $V_{\mathrm{DS}}=30 \mathrm{mV}$ (inset: the noise spectra density measured at different $V_{\mathrm{DS}}$ and $V_{G}=0 \mathrm{~V}$ before irradiation); (b) measured at $V_{\mathrm{DS}}$ $=30 \mathrm{mV}$ and different $V_{G}$ before gamma radiation treatment; and (c) measured at $V_{\mathrm{DS}}=30 \mathrm{mV}$ and different $V_{G}$ after gamma radiation treatment with a dose of $1 \times 10^{6} \mathrm{rad}$.

mation about the processes and mechanisms of transport formation in the structure. An analysis of the current noise power spectra allows us to confirm that the main noise source is caused by transport phenomena in CNTs as will be shown below.

As in the case of output characteristics, the resistance of CNTs increases by about $25 \%$ after $1 \times 10^{6} \mathrm{rad}$ irradiation in comparison with the resistance before gamma treatment. The results of noise measurements are shown in Fig. 3 and they correlate with transport results. The level of normalized current noise power spectra was found to be independent of drain and gate voltages [Fig. 3(a)]. This fact confirms that the main source of noise in the structure is related to the transport phenomena in the region between the contacts of the structure and that contact noise contribution can be neglected. At the same time, after normalization to frequency, $f$, small changes of the noise level in the high-frequency region of the spectra can be found [Fig. 3(b)]. After gamma irradiation this flicker noise component shifts to the lower frequency range and demonstrates decreased changes with $V_{G}$. It is known that the relative noise level in different kinds of materials can be estimated according to the Hooge relation as follows:

$$
\frac{f S_{I}}{I^{2}}=\frac{\alpha_{H}}{N},
$$

where $\alpha_{H}$ is the Hooge parameter, $N$ is the total number of carriers in the system, $f$ is the frequency, $S_{I}$ is the current noise spectral density, and $I$ is the current.
The substitution of the total number of carriers that can be found as $N=L_{\mathrm{DS}}^{2} / e \mu R_{N}$ into Eq. (4) yields

$$
\alpha_{H}=\frac{f S_{I} L_{\mathrm{DS}}^{2}}{I^{2} e \mu R_{N}},
$$

where $e$ is the electron charge and $R_{N}$ is the resistance of CNTs.

If the current $I$ drops due to the mobility $\mu$ decreasing then value of $f S_{I} / I^{2}$ increases 1.3 times and this results in the increase in $\alpha_{H}$. In the spectra of the low-frequency range, the noise level at $f=1 \mathrm{~Hz}$ also increases by about 1.3 times. This fact confirms the suggestion that the noise in CNT is caused by defects introduced by gamma radiation. Taking into account the measured value of mobility $1.5 \times 10^{5} \mathrm{~cm}^{2} / \mathrm{V} \mathrm{s}$ and Eq. (5), one can obtain the Hooge parameter as $\alpha_{H}=3.8$ $\times 10^{-3}$ before gamma irradiation and $5.3 \times 10^{-3}$ after irradiation. This low value of the parameter is even better than the values obtained previously ${ }^{16,17}$ for CNT samples with a long length of the tube without gamma irradiation treatment and is comparable with those of conventional semiconductors such as $\mathrm{GaAs}, \mathrm{GaN}$, and $\mathrm{Si}$, which indicates the low noise level of the CNTs themselves.

We found for the $1 / f$ noise component (in pure form resolved at $19 \mathrm{kHz}$ ) from $f S_{I} / I^{2}$ that the dependence on gate voltage at increasing $V_{G}$ from -2 to $+2 \mathrm{~V}$ noise level increases 1.25 times and the current decreases 1.11 times. Such noise behavior is approximately the same before and after gamma irradiation. According to Eq. (5), the value $f S_{I} / I^{2}$ increased with decreasing current as $1 / I$ in 1.11 times. The remaining 1.13 times can be explained by decreasing in $\sqrt{1.13}=1.06$ times the active part of the length of the channel. The latter is usually observed in FETs and confirms that the origin of $1 / f$ noise is not due to the contact noise.

In addition to the flicker noise component, the generation-recombination (GR) noise component can be resolved in the low-frequency range of the spectra.

The GR component of the noise spectra can be described by

$$
\frac{f S_{I}^{\mathrm{GR}}}{I^{2}}=\frac{f S_{I}^{\mathrm{GR}}(0)}{1+(2 \pi f \tau)^{2}},
$$

where $S_{I}^{\mathrm{GR}}$ is the GR current noise spectral density and $\tau$ is the time constant of the GR process.

From this part of noise spectra, the numerator increases twofold after gamma irradiation with respect to noise level before irradiation, at the same time the denominator change is caused by decreasing time constant $\tau$ as a result of the influence of radiation. It should be noted that the GR component has weak dependence on gate voltage but the dependence is nonmonotonous in both cases before as well as after gamma irradiation. The noise spectra after subtracting the $1 / f$ noise component are shown in Fig. 4(a) (before gamma irradiation) and Fig. 4(b) (after irradiation). The main difference between the spectra is the shift in regions with $1 / f^{2}$ dependence to the higher frequency range. The result indicates that traps with different energy levels were activated as a device was exposed to small doses of gamma radiation. At the same time, the noise level has approximately the same 

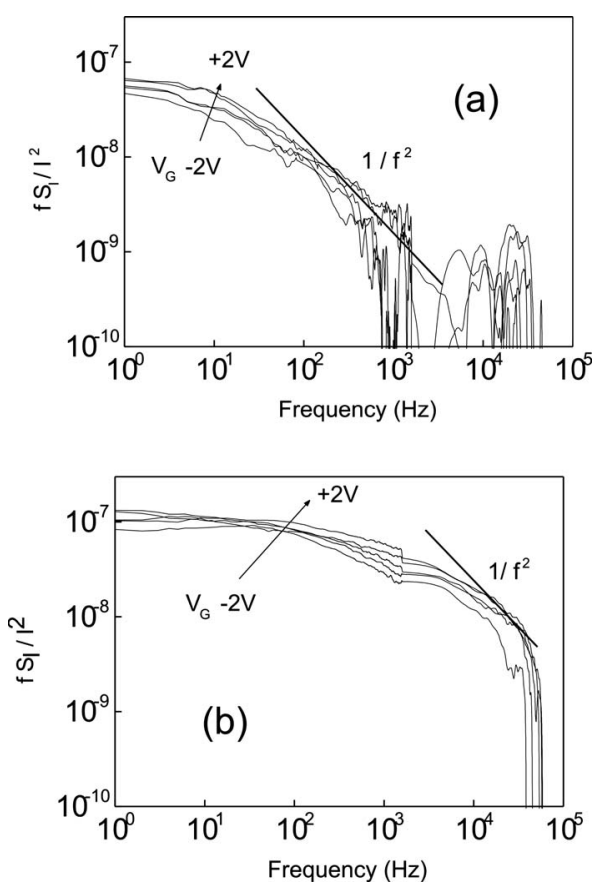

FIG. 4. Normalized current noise power spectra of CNT-FETs: (a) measured at different $V_{G}$ before gamma radiation treatment, plotted after subtraction of the $1 / f$ noise component; (b) measured at different $V_{G}$ after gamma radiation treatment with a dose of $1 \times 10^{6} \mathrm{rad}$, plotted after subtraction of the $1 / f$ noise component.

value for both cases and the concentration of the traps does not change very much at this low exposure dose.

In addition, the results of transport and noise measurements obtained after gamma radiation treatment with a dose of $2 \times 10^{6}$ rad confirm such behavior. Figure 5(a) shows the transfer characteristics of the CNT-FET measured after such a dose of gamma irradiation. The I-V characteristics demonstrate an additional decrease in the total resistance which is even stronger than in the case of $1 \times 10^{6} \mathrm{rad}$ gamma radiation dose. With an increase in the radiation dose to 2 $\times 10^{6} \mathrm{rad}$ the threshold voltage is reduced with improved on/off current ratio. The value of the maximum transconductance is found to be $1.7 \mu \mathrm{S}$ and only decreases slightly in comparison to $g_{m}$ before gamma radiation treatment. The transfer characteristics of CNT-FETs demonstrate that the current through the structure can be effectively controlled in the region to nearly $V_{G}=0$ by the gate voltage, also after gamma radiation treatment. The region around zero gate voltage with only plus and minus $1 \mathrm{~V}$ voltage applied to the gate is ideal for different kinds of applications.

The radiation also does not have any significant influence on the noise properties [Fig. 5(b)], which proves the radiation hardness of the nanotubes. The noise behavior follows the flicker $\left(\sim 1 / f^{\alpha}\right)$ noise with slope $\alpha$ equal to one [Fig. 5(b)]. The Hooge parameter obtained after radiation dose of $2 \times 10^{6} \mathrm{rad}$ was found to be $\alpha_{H}=2.4 \times 10^{-2}$. The magnitude of the $1 / f$ noise increased as $I_{\mathrm{DS}}^{2}$ with drainsource current up to $50 \mu \mathrm{A}$, as shown in inset to Fig. 5(b).

The level of normalized current noise power spectra was also found to be independent on drain and gate voltages. These experiments rule out any predominant contribution
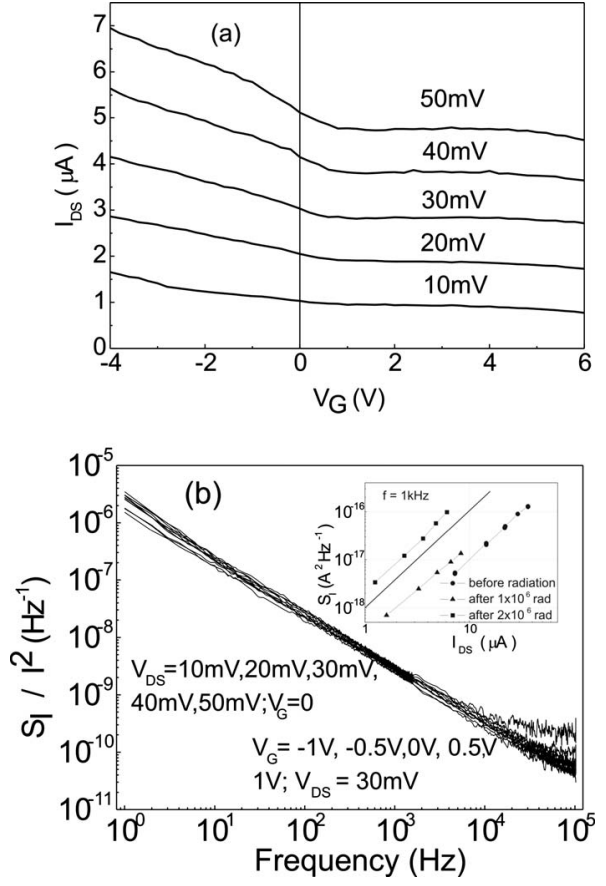

FIG. 5. (a) Transfer characteristics of CNT-FETs measured at different $V_{\mathrm{DS}}$ after gamma radiation treatment with a dose of $2 \times 10^{6} \mathrm{rad}$ and (b) normalized current noise power spectra of CNT-FETs measured at different $V_{G}$ and constant $V_{\mathrm{DS}}$ and vice versa before and after gamma radiation treatment with a dose of $1 \times 10^{6}$ and $2 \times 10^{6} \mathrm{rad}$; (inset) current noise power spectra of CNT-FETs plotted as a function of $I_{\mathrm{DS}}$.

from the contacts and confirm that the main source of noise in the structure is related to the transport phenomena in CNTs.

\section{CONCLUSION}

In summary, our analysis shows that the difference in output characteristics of the FETs before and after small doses of gamma radiation treatment is due to a reduced contribution to the total current parallel to the nanotube resistance. The transconductance of the CNT-FETs and level of noise did not change strongly after $\gamma$-irradiation at doses of $1 \times 10^{6}$ and $2 \times 10^{6} \mathrm{rad}$. The results show that active $\gamma$-radiation treatment can improve the transport and noise properties of CNT-FETs at some small optimal dose. The radiation treatment was found to decrease the influence of parasitic conduction channels on dc characteristics of the device. The Hooge parameters obtained are comparable with typical values obtained for conventional semiconductors.

The obtained results should be taken into account for the development of future chemical and biological sensors.

\footnotetext{
${ }^{1}$ Nanotubes and Nanowires, edited by P. J. Burke (World Scientific, Singapore, 2007).

${ }^{2}$ S. Heinze, J. Tersoff, R. Martel, V. Derycke, J. Appenzeller, and $\mathrm{Ph}$. Avouris, Phys. Rev. Lett. 89, 106801 (2002).

${ }^{3}$ Z. H. Chen, J. Appelzeller, J. Knoch, Y. M. Lin, and P. Avouris, Nano Lett. 5, 1497 (2005).

${ }^{4}$ J. Guo, Yu. Li, Sh. Wu, and W. Li, Nanotechnology 16, 2385 (2005).

${ }^{5}$ V. Skakalova, U. Dettlaff-Weglikowska, and S. Roth, Diamond Relat. Mater. 13, 296 (2004).

${ }^{6}$ W.-T. Wu, L. Shi, Yu. Wang, W. Pang, and Q. Zhu, Nanotechnology 19, 125607 (2008).

${ }^{7}$ A. E. Belyaev, J. Breza, E. F. Venger, M. Vesely, I. Yu. Il'in, R. V. Kona-
} 
kova, J. Linday, V. G. Lyapin, V. V. Milenin, I. V. Prokopenko, and Yu. A. Thorik, Radiation Resistance of GaAs-based Microwave Schottky-Barrier Devices (Interpress, Kiev, 1998).

${ }^{8}$ A. M. Kurakin, S. A. Vitusevich, S. V. Danylyuk, H. Hardtdegen, N. Klein, Z. Bougrioua, B. A. Danilchenko, R. V. Konakova, and A. E. Belyaev, J. Appl. Phys. 103, 083707 (2008).

${ }^{9}$ A. Ural, Y. Li, and H. Dai, Appl. Phys. Lett. 81, 3464 (2002).

${ }^{10}$ A. Behnam, G. Bosman, and A. Ural, Phys. Rev. B 78, 085431 (2008).

${ }^{11}$ S. M. Sze, Physics of Semiconductor Devices (Wiley, New York, 1981).

${ }^{12}$ F. B. McLean, H. E. Boesch, Jr., and T. R. Oldham, Ionizing Radiation
Effects in MOS Devices and Circuits, (Wiley, New York, 1989).

${ }^{13}$ M. Yoshikawa, H. Itoh, Y. Morita, I. Nashiyama, S. Misawa, H. Okumura, and S. Yoshida, J. Appl. Phys. 70, 1309 (1991).

${ }^{14}$ C. T. Sah, IEEE Trans. Nucl. Sci. 23, 1563 (1976).

${ }^{15}$ J. R. Schwank, M. R. Shaneyfelt, D. M. Fleetwood, J. A. Felix, P. E. Dodd, Ph. Paillet, and V. Ferlet-Cavrois, IEEE Trans. Nucl. Sci. 55, 1833 (2008).

${ }^{16}$ F. N. Hooge, Phys. Lett. A 29, 139 (1969).

${ }^{17}$ M. Ishigami, J. H. Chen, E. D. Williams, D. Tobias, Y. F. Chen, and M. S. Fuhrer, Appl. Phys. Lett. 88, 203116 (2006). 